\title{
sciendo
}

\section{Hormonal Response to Incremental and Continuous Exercise in Cyclists with Left Ventricle Hypertrophy}

\author{
by

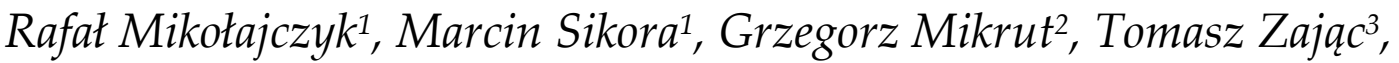 \\ Aleksandra Żebrowska ${ }^{4}$
}

The aim of this study was to assess the effects of incremental and continuous exercise on the concentration of insulin-like growth factor-1 (IGF-1), growth hormone (GH), testosterone (T), and cortisol (C), as well as to investigate whether increased cardiac dimensions in cyclists were related to changes in these hormones and cardiac biomarkers. The study included 30 elite cyclists divided into two groups, i.e., athletes with left ventricle hypertrophy (a LVH group), and a control group (CG) without LVH. The study protocol included performance of a standard incremental exercise (IncEx) test to measure athletes' maximum power (Pmax), maximum oxygen uptake (VO2max), and lactate threshold (LAT). The IncEx test results were then used to determine the intensity of the continuous exercise (ConEx) test which was performed after the 1-week washout period. Cyclists with LVH and without LVH did not differ in resting hormone concentrations and cardiac biomarkers levels. There was a significant effect of exercise on serum IGF-1 levels $(p<0.05)$ in the LVH group and a combined effect of the type of exercise and LVH on IGF-1 $(p<0.05)$. Cyclists with LVH demonstrated higher post exercise $T$ levels recorded in response to exercise compared to the $C G(p<0.01)$. Significantly higher serum T levels were observed in response to ConEx compared to IncEx in the LVH group and the CG $(p<0.05$ and $p<0.05$, respectively). In the LVH group, a significant positive correlation between the post-exercise T/C ratio and left ventricular mass index was observed $(r=0.98, p<0.01)$. There were no effects of heart hypertrophy on cardiac standard biomarkers. Incremental and continuous exercise caused a marked increase in steroid hormone concentrations and moderate strengthening of insulin growth factors effects. Regular incremental exercise seems to induce beneficial cardiac adaptations via significant increases in the concentration of anabolic factors compared to the same training mode yet with constant exercise intensity.

Key words: hormones, cardiac markers, myocardium, cycling, endurance exercise.

\section{Introduction}

Long term exercise causes chronic adaptive changes of the heart, including hypertrophy, especially an increase in the left ventricular mass and wall thickness, which in turn is associated with increased physical endurance (Pellicia et al. 2018). Several factors determine cardiac hypertrophy in competitive athletes, such as the intensity and duration of exercise, the training mode (Utomi et al., 2013) and training experience (Sharma, 2003). It is well established that different sports activities leading to continuous dynamic or static exercise effort can induce different discipline-specific levels of heart hypertrophy (Maillet et al., 2013; Pellicia et al., 2018).

Signals that trigger cardiac muscle hypertrophy include higher gene expression and increased secretion of several growth factors

\footnotetext{
1 - Department of Physiological and Medical Sciences, Academy of Physical Education, Katowice, Poland.

2 - Department of Sport and Tourism Management, Academy of Physical Education, Katowice, Poland;.

3 - Human Performance Laboratory, Academy of Physical Education, Katowice, Poland.

4 - Institute of Sport Sciences, Academy of Physical Education, Katowice, Poland.
} 
during physical effort (Pelliccia and Thompson, 2006; Vega et al., 2018). The insulin-like growth factor phosphoinositide 3-kinase (PI3K)/Akt/glycogen synthase 3 (GSK-3) pathway is among the major signaling molecules governing cardiac hypertrophy response (Huang et al., 2003; Kim et al., 2008). Exercise induced myocardial expression of IGF-1 leads to cardiac hypertrophy after long-term training (Scheinowitz et al., 2003). In a human study, hemodynamic overload of the heart during incremental exercise was associated with enhanced cardiac IGF-1 formation (Neri Serneri et al., 2001), improved left ventricular function and exercise tolerance (Żebrowska et al., 2009). IGF-1 is secreted mainly by the liver in response to growth hormone $(\mathrm{GH})$ stimulation, which is called the GH-IGF-1 axis (Eliakim et al., 2010; Troncosco et al., 2014).

High-intensity exercise has been known to increase the circulating IGF-1 and/or GH-IGF-1 axis in both untrained and trained subjects (Schwarz et al. 1996). Some evidence suggests that endurance training is associated with decreased blood testosterone (T) and increased cortisol (C) concentrations, which might potentially influence protein synthesis and degradation, thereby affecting the GH/IGF-I axis response (Mettauer et al., 2001). It has been hypothesized that testosterone induces a reduction in circulating IGF-1 levels and may have a direct, IGF-1 and $\mathrm{GH}$-independent effect on cardiac growth (Zung et al., 1999). In line with the involvement of the baseline levels of these hormones in physiological cardiac remodeling there are data obtained in animal studies (Huang et al., 2003; Żebrowska et al., 2017), endurance trained athletes (Eliakim et al., 2010), and healthy adolescents (Rundqvist et al., 2019).

In contrast, relatively little is known concerning the hormonal changes that occur following high intensity incremental or moderate continuous intensity exercise and its implication on the potential cardiac remodeling in cyclists (Nishimura et al., 1980). We hypothesized that since endocrine responses in cyclists with $\mathrm{LVH}$ might depend on exercise intensity and duration, independently of hemodynamic conditions, exercise-induced insulin growth factors and anabolic hormone levels should be higher than in cyclists with a normal left ventricle dimension.

A few reports have also suggested that participation in ultra-endurance cycling events is associated with significant negative changes in cardiac function (LeGoff et al., 2015; Serrano Ostariz et al., 2013), including altered left and right ventricular functions, the appearance of wall motion abnormalities and increases in cardiac biomarker levels (Carranza-Garcia et al., 2011).

Considering these potentially negative effects on the heart, it is unclear whether there is an association between the echocardiographic indicators of heart structure, cardiac biomarkers and exercise induced concentration of selected anabolic and catabolic hormones. Therefore, the aim of this study was to assess the effects of incremental and continuous exercise on the concentration of insulin-like growth factor-1 (IGF$1)$, growth hormone $(\mathrm{GH})$, testosterone $(\mathrm{T})$, and cortisol (C), as well as to investigate whether increased cardiac dimensions in cyclists were related to these hormones and the concentration of cardiac biomarkers.

\section{Methods}

\section{Participants}

The study included 30 elite cyclists recruited from sports associations in Poland. They were divided into groups based on a clinical finding of left ventricle hypertrophy (LVH), i.e., a LVH group and a control group (CG) without LVH. Participants were excluded if they had a concurrent cardiovascular or respiratory disorder. Cyclists' body mass and body composition were determined using bioelectrical impedance analysis (BIA; InBody Data Management System, Biospace, Korea). Cyclists from both groups were characterized by similar body mass and training status (Table 1). All individuals were instructed to abstain from strenuous exercise $24 \mathrm{~h}$ prior to the study. No caffeine, antioxidants supplements or alcohol were permitted during $48 \mathrm{~h}$ before the experiment. The study was approved by the local Bioethical Committee (University Ethics Committee decision N3/2010) and conducted in accordance with the Declaration of Helsinki of the World Medical Association.

\section{Study protocol}

The study protocol included two visits to the laboratory. During the first visit, all cyclists performed a standard incremental exercise (IncEx) test to measure their individual aerobic capacity (Sport Excalibur, the Lode Company, The 
Netherlands) in terms of maximum power $\left(\mathrm{P}_{\max }\right)$, maximum oxygen uptake $\left(\mathrm{VO}_{2 \max }\right)$, and lactate threshold (LAT). Prior to the exercise test, all participants underwent baseline echocardiography. A resting echocardiographic examination was performed in all participants in the morning after a night's rest (Rest). At baseline, the IncEx tests were used to determine the intensity of the continuous exercise test (ConEx). After a 1-week washout period all participants performed the ConEx test.

\section{Echocardiography}

Echocardiography was performed with a GE Vivid E9 device (General Electric, Horten, Norway), with a head frequency of $2.5 \mathrm{MHz}$. The resting echocardiogram was performed using the one-dimensional (M-mode), two-dimensional (2D), and Doppler methods.

From the M-mode image obtained in the parasternal view under the control of the twodimensional mode, primary measurements were made as follows: left ventricular end-diastolic diameter (LVEDd), left ventricular end-systolic diameter (LVESd), interventricular septum thickness (IVS), and posterior wall thickness (PWT) of the left ventricle. Mean values of three cardiac cycles were analyzed. On the basis of the obtained measurements, left ventricular mass (LVM) and left ventricular muscle mass index (LVMI) were calculated (Devereux, 1977). To assess cardiac hypertrophy, LVMI values above $134 \mathrm{~g} / \mathrm{m}^{2}$ were adopted, as recommended by Levy et al. (1987). Left ventricular (LV) systolic function was determined by measurements of the left ventricular ejection fraction (LVEF). The intraobserver coefficients of variation were $2.5 \%$ for measurements of LV diameters, and $3.6 \%$ for measurements of the LVEF.

\section{Exercise protocol}

All cyclists performed an incremental exercise (IncEx) test on a cycling ergometer (Sport Excalibur, the Lode Company, The Netherlands) to determine individual exercise performance and maximum oxygen uptake $\left(\mathrm{VO}_{2 \max }\right)$. Participants started pedaling without any load for $3 \mathrm{~min}$ at a frequency of $60-70 \mathrm{rpm}$, and then the load was increased every $3 \mathrm{~min}$ by $40 \mathrm{~W}$ until the maximum individual load was reached. Criteria for reaching of $\mathrm{VO}_{2 \max }$ were voluntary exhaustion, the respiratory ratio equal to or exceeding 1.15 (RER 21.1), a $\mathrm{VO}_{2}$ plateau and blood lactate concentration $\geq 8.0 \mathrm{mmol} / \mathrm{L}$. The heart rate $(\mathrm{HR})$, oxygen uptake $\left(\mathrm{VO}_{2}\right)$, carbon dioxide output $\left(\mathrm{VCO}_{2}\right)$, and respiratory minute volume $\left(\mathrm{V}_{\mathrm{E}}\right)$ were continuously monitored before and during the exercise test. Breathing variables were measured with the Oxycon RALPHA (Jaeger, Germany). HR (PE-3000 Sport-tester, Polar Inc., Finland) and systolic and diastolic blood pressures (SDB/DBP) were measured (HEM-907 XL, Omron Corporation, Japan) before and immediately after the test (HEM-907 XL, Omron Corporation, Japan).

One week later all cyclists performed a continuous exercise test (ConEx). After a $5 \mathrm{~min}$ warm-up, cyclists performed a 60-min ConEx test with the intensity of the lactate threshold ( $D_{\max }$ method) (LAT) calculated individually for each athlete during the IncEx test.

\section{Biochemical assays}

Blood hormone concentration assessment was performed at rest before both exercise tests (Rest), immediately afterwards (Max), and $15 \mathrm{~min}$ into recovery (15 min Post-Ex). The concentration insulin-like growth factor-1 (IGF-1) was determined with the IGF1-RIACT kit (Cisbio, France) and growth hormone (GH) levels were determined with the hGH-RIACT kit (Cisbio, France). The insulin-like growth factor-binding protein 3 (IGFBP-3) concentration was also assessed with the IRMA method using the IGFBP3-IRMA-CT KIPB1014 kit (Biosource Europe SA Company, England). The concentrations of testosterone $(\mathrm{T})$, free testosterone (freeT), and cortisol (C) in blood serum were determined by radioimmunoassay (RIA) using TESTO-CT-2 (Cisbio, France) and CORT-CT-2 (Cisbio, France) kits per manufacturer's instructions.

Samples of venous blood for cardiac biomarker assessment were taken at rest before both exercise tests (Rest) and immediately afterwards (Max). N-terminal pro-brain-type natriuretic peptide (NT-proBNP) levels were assayed using the immunoassays method (SEA485Hu 96 Tests, Cloud-Clone Corporation, Katy, TX, USA). The measurements of cardiac troponin $\mathrm{T}$ (cTnT) were performed using immunoassays (SED22HU 96 Tests, Cloud-Clone Corporation, Houston, TX, USA). Serum activities of creatine kinase myocardial band (CK-MB) were evaluated using the immune-inhibition method (BioMaxima, Lublin, Poland). Concentrations of 
ischemic modified albumin (IMA) were determined by the immune-enzymatic method (CUSABIO BIOTECH CO., LTD, Wuhan, China). The heart fatty acid-binding protein (H-FABP) levels were determined using the immuneenzymatic method (Human H-FABP HK401 Elisa Kit, Hycult Biotech, Uden, The Netherlands).

Blood lactate concentrations (LA) were determined using the Biosen $C_{-}$line method (EKF Diagnostic $\mathrm{GmbH}$, Germany); blood gases and acid-base balance were also analyzed (RapidLab 348; Bayer Diagnostics, Germany). To avoid the effects of diurnal variations blood samples were taken in the morning at the same time (between 8.00 and $9.00 \mathrm{am})$. The degree of hemoconcentration (\%) was calculated according to the formula of subtracting the peak hematocrit from the minimum hematocrit recorded and multiplying by 100; all biochemical variables were corrected according to plasma volume.

\section{Statistical analysis}

All data are presented as the mean and standard deviation (SD). The total sample size was calculated using the Altman nomogram and alfa value of 0.05 for 0.07 test power. Verifications of the differences between analyzed variables (pre exercise vs. post exercise), type of exercise (IncEx vs. ConEx), and groups (LVH vs. CG) were carried out using three-way ANOVA with correction for repeated measures. Homogeneity of variance was verified by the Levene's test. In the absence of homogeneity the Kruskal-Wallis test was used. The significance of differences between the variables was verified with a post-hoc Tukey test. Correlation coefficients between all the variables were determined with the Pearson's rank order test. Microsoft Excel 2007 (Microsoft Corp., Washington, USA) and the Statistics Package v.12 (StatSoft Poland, 12.0) were used for data processing and analyses. The differences were considered statistically significant at $p<0.05$.

\section{Results}

Participants' characteristics and echocardiography

Somatic variables, training status and physical performance characteristics of the study participants are presented in Table 1. There were no significant differences in somatic variables, training status, and maximum oxygen uptake $\left(\mathrm{VO}_{2 \max }\right)$ between cyclists with $\mathrm{LVH}$ and the CG $(p>0.05)$.
Per inclusion criteria, athletes with LVH had a significantly higher $\operatorname{LVM}(\mathrm{F}=40.1, p<0.001)$ and LVMI $(F=50.3, p<0.001)$ in comparison with the CG group. Higher values of IVS and PWT were reported in the $\mathrm{LVH}$ group relative to the $\mathrm{CG}(\mathrm{F}=$ $15.2, p<0.001$ and $\mathrm{F}=15.2 p<0.001$, respectively) (Table 2). The analysis of variance showed a significant effect of LVH $(\mathrm{F}=11.8 ; p<0.001)$ on LVEDd, which was larger in the LVH group than in the CG. All resting ECG were normal. No significant differences were found in LVESd, LVEF, SV, resting HR, and SBP/DBP between cyclists with LVH and without LVH.

Serum hormone concentrations in response to incremental and continuous exercises

Participants did not significantly differ in resting hormone concentrations before the IncEx and ConEx tests. ANOVA revealed significant effect of IncEx on serum IGF-1 levels ( $\mathrm{F}=3.6, p<$ 0.05 ) in the LVH group (Table 3). There was a significant combined effect of the type of exercise and LVH on IGF-1 ( $\mathrm{F}=5.2, p<0.05)$ with a tendency to a higher post-exercise IGF-1 level after IncEx compared to ConEx. No significant differences in both exercise protocols were found for IGF-1, IGFBP-3, and IGF-1/IGFBP-3 ratio between cyclists with and without LVH. Serum GH levels were increased in response to incremental and continuous exercise protocols in comparison to resting levels in both groups, i.e., cyclists with LVH and the CG (Figure 1). Despite the lack of significant effect of LVH on GH, the significant impact of the type of exercise on serum GH levels $(\mathrm{F}=24.8, \quad p<0.001)$ was observed. ANOVA revealed significant effect of IncEx and ConEx on serum $\mathrm{T}$ levels $(\mathrm{F}=9.3 ; p<0.01, \mathrm{~F}=11.4$; $p<0.01$, respectively) in cyclists with $\mathrm{LVH}$ and the combined influence of $\mathrm{LVH}$ and incremental exercise on serum $\mathrm{T}$ levels $(\mathrm{F}=3.00 ; p<0.05)$. Cyclists with LVH demonstrated higher postexercise testosterone levels recorded in response to IncEx compared to the CG ( $\mathrm{F}=8.2, p<0.01)$. Significantly higher serum $\mathrm{T}$ levels were observed in response to ConEx compared to IncEx in both the CG and the LVH group ( $p<0.05$ and $p<0.05$, respectively) (Figure 2). Cyclists with $\mathrm{LVH}$ demonstrated a non-significant tendency for higher freeT levels at maximal IncEx and during recovery (15 min Post-Ex) than athletes without cardiac hypertrophy (Tables 3 and 4).

Significant effect of IncEx and ConEx was 
observed in serum C levels. Significant inter group differences (LVH vs. CG) regarding $\mathrm{C}$ levels were found at maximal exercise intensity ( $\mathrm{F}$ $=12.3, p<0.001)$ in the ConEx test. Moreover, significantly lower $15 \mathrm{~min}$ post exercise $C$ levels in the LVH group compared to the CG in response to IncEx $(p<0.05)$ were observed (Tables 3 and 4$)$. No significant combined effects of LVH and the type of exercise were observed in $C$ levels and the $\mathrm{T} / \mathrm{C}$ ratio. There was a significant positive correlation between LVMI and the post exercise $\mathrm{T} / \mathrm{C}$ ratio $(\mathrm{r}=0.98, p<0.01)$ in the LVH group and also between freeT levels and LVM $(r=0.57, p<$ 0.05).

Incremental and continuous exercise tests had little effect of serum cardiac biomarkers except lower post exercise H-FABP levels $(p<0.05)$ compared to baseline levels in ConEx in the LVH group compared to the CG. ANOVA revealed no significant effect of the type of exercise, $\mathrm{LVH}$ and exercise on post exercise cardiac biomarker levels $(p>0.05)$ (Table 5). Participants showed no statistically significant correlations between serum cardiac biomarker concentrations, hormonal levels and echocardiography variables.

Table 1

Characteristics of study participants: cyclists with left ventricle hypertrophy (LVH) and without left ventricle hypertrophy (CG).

\begin{tabular}{|c|c|c|c|c|}
\hline Variable & CG (n=16) & $\mathrm{LVH}(\mathrm{n}=14)$ & F & $p$ \\
\hline Age [years] & $26.3(11.8)$ & $24.4(3.5)$ & 0.3 & 0.57 \\
\hline Body height [cm] & $177.7(3.4)$ & $179.4(7.3)$ & 0.7 & 0.40 \\
\hline Body mass $[\mathrm{kg}]$ & $71.9(6.6)$ & $71.9(7.3)$ & 0.0 & 0.99 \\
\hline $\mathrm{BSA}\left[\mathrm{m}^{2}\right]$ & $1.9(0.1)$ & $1.9(0.1)$ & 0.1 & 0.77 \\
\hline BMI $\left[\mathrm{kg} / \mathrm{m}^{2}\right]$ & $22.7(2.1)$ & $22.4(2.0)$ & 0.2 & 0.64 \\
\hline FM [kg] & $9.1(3.7)$ & $8.6(2.7)$ & 0.2 & 0.64 \\
\hline FFM $[\mathrm{kg}]$ & $62.2(5.0)$ & $66.0(6.3)$ & 3.1 & 0.09 \\
\hline TBW $[\mathrm{kg}]$ & $45.6(3.6)$ & $48.3(4.6)$ & 3.2 & 0.08 \\
\hline $\mathrm{VO}_{2 \max }[\mathrm{ml} / \mathrm{kg} / \mathrm{min}]$ & $61.1(7.6)$ & $62.0(7.5)$ & 0.1 & 0.77 \\
\hline $\begin{array}{c}\text { Training status } \\
\text { [years] }\end{array}$ & $9.1(4.3)$ & $9.9(5.1)$ & 1.1 & 0.57 \\
\hline Powermax [Watt] & 366.1 (48.6) & $391.4(54.7)$ & 1.6 & 0.22 \\
\hline
\end{tabular}

$B S A$ - body surface area; BMI - body mass index; FM - fat mass; FFM - fat free mass; $T B W$ - total body water; $V O_{2 m a x}$ - maximal oxygen uptake. 


\begin{tabular}{|c|c|c|c|c|}
\hline \multicolumn{5}{|c|}{$\begin{array}{l}\text { Echocardiographic variables and blood pressure in cyclists with left } \\
\text { ventricle hypertrophy }(\mathrm{LVH}) \text { and without left ventricle hypertrophy (CG) }\end{array}$} \\
\hline Variable & CG (N=16) & LVH $(\mathrm{N}=14)$ & $\mathrm{F}$ & $p$ \\
\hline LVM [g] & 193.8 (31.5) & $301.3(58.4)$ & 40.1 & 0.00 \\
\hline LVMI $\left[\mathrm{g} / \mathrm{m}^{2}\right]$ & $102.5(16.3)$ & $158.0(25.6)$ & 50.3 & 0.00 \\
\hline IVS [mm] & $10.1(1.8)$ & $12.1(0.6)$ & 15.2 & 0.00 \\
\hline PWT [mm] & $9.1(1.5)$ & $11.0(1.1)$ & 15.2 & 0.00 \\
\hline LVEDd [mm] & $49.0(3.6)$ & $53.9(4.1)$ & 11.8 & 0.00 \\
\hline LVESd [mm] & $30.5(2.9)$ & $31.0(3.5)$ & 0.2 & 0.68 \\
\hline LVEF\% & $60.4(4.9)$ & $60.6(4.2)$ & 0.0 & 0.94 \\
\hline $\mathrm{SV}[\mathrm{mL}]$ & $86.0(12)$ & $94.4(10.1)$ & 2.0 & 0.17 \\
\hline $\mathrm{HR}_{\text {Rest }}[\mathrm{b} / \mathrm{min}]$ & $73.5(10.9)$ & $70.1(9.8)$ & 0.4 & 0.54 \\
\hline $\mathrm{HR}_{\operatorname{Max}}[\mathrm{b} / \mathrm{min}]$ & $181.3(13.1)$ & $192.4(8.4)$ & 4.0 & 0.07 \\
\hline SBPRest $[\mathrm{mmHg}]$ & $119.7(12.9)$ & $124.6(14.1)$ & 1.0 & 0.33 \\
\hline $\mathrm{DBP}_{\text {Rest }}[\mathrm{mmHg}]$ & $79.4(11.3)$ & $77.5(11.2)$ & 0.2 & 0.66 \\
\hline
\end{tabular}

LVM - left ventricular mass; LVEDd - left ventricular end-diastolic diameter; LVESd - left ventricular end-systolic diameter; LVEF - left ventricular ejection fraction; $S V$-stroke volume; $H R_{\text {Rest }}$ - resting heart rate; $S B P_{\text {Rest }}$ - systolic blood pressure at rest; $D B P_{\text {Rest }}$ - diastolic blood pressure at rest.

Table 3

Effects of incremental exercise (IncEx) on hormone concentrations in cyclists with left ventricle hypertrophy (LVH) and without left ventricle hypertrophy (CG).

\begin{tabular}{ccccccccc}
\hline \multicolumn{7}{c}{ Incremental exercise test (IncEx) } \\
\hline Variables & \multicolumn{7}{c}{ Rest } & \multicolumn{7}{c}{ Max min Post-Ex } & F & $p$ \\
& Mean & SD & Mean & SD & Mean & SD & & \\
\hline T [nmol/1] & 30.1 & 12.1 & 37.3 & 16.0 & 40.7 & 17.5 & 9.3 & 0.00 \\
free T [nmol/1] & 11.9 & 3.9 & 12.1 & 2.8 & 11.4 & 2.9 & 0.3 & 0.7 \\
C [nmol/1] & 442.5 & 186.1 & 548.1 & 295.9 & 579.8 & 270.9 & 3.4 & 0.05 \\
GH [ng/ml] & 3.5 & 4.5 & 44.8 & 24.3 & 39.2 & 18.1 & 40,5 & 0.00 \\
IGF-1 [ng/ml] & 288.8 & 70.8 & 312.8 & 73.1 & 284.7 & 68.3 & 3.6 & 0.03 \\
IGFBP-3 [ng/ml] & 3088.5 & 1167.5 & 3734.8 & 1429.0 & 3494.9 & 1567.1 & 1.7 & 0.20 \\
& & & & & & & & \\
T [nmol/1] & 25.6 & 12.3 & 32.0 & 12.7 & 30.5 & 15.7 & 5.2 & 0.01 \\
free T [nmol/1] & 10.1 & 1.3 & 11.3 & 1.0 & 10.3 & 0.7 & 1.3 & 0.3 \\
C [nmol/1] & 549.1 & 205.7 & 645.3 & 204.2 & 789.3 & 237.4 & 7.8 & 0.02 \\
GH [ng/ml] & 2.6 & 2.4 & 54.6 & 33.4 & 44.0 & 24.9 & 26.6 & 0.00 \\
IGF-1 [ng/ml] & 289.8 & 119.2 & 333.3 & 146.0 & 304.0 & 119.4 & 3.6 & 0.03 \\
IGFBP-3 [ng/ml] & 2370.3 & 1017.9 & 2967.3 & 1441.6 & 3497.0 & 2617.5 & 1.2 & 0.33 \\
\hline
\end{tabular}




\section{Table 4}

Effects of continuous exercise (ConEx) on hormone concentrations in cyclists with left ventricle hypertrophy (LVH) and cyclists without left ventricle hypertrophy (CG).

\begin{tabular}{|c|c|c|c|c|c|c|c|c|}
\hline \multicolumn{9}{|c|}{ Continuous exercise (ConEx) } \\
\hline \multirow{3}{*}{ Variables } & \multicolumn{2}{|c|}{ Rest } & \multicolumn{2}{|c|}{ Max } & \multicolumn{2}{|c|}{15 min Post-Ex } & \multirow{3}{*}{ F } & \multirow{3}{*}{$p$} \\
\hline & Mean & SD & Mean & SD & Mean & SD & & \\
\hline & \multicolumn{6}{|c|}{ Cyclists with LVH } & & \\
\hline $\mathrm{T}[\mathrm{nmol} / \mathrm{l}]$ & 30.2 & 12.2 & 48.4 & 14.2 & 42.1 & 17.7 & 11.42 & 0.01 \\
\hline free $\mathrm{T}[\mathrm{nmol} / \mathrm{l}]$ & 8.2 & 3.1 & 10.6 & 4.6 & 10.6 & 5.0 & 2.6 & 0.13 \\
\hline $\mathrm{C}[\mathrm{nmol} / \mathrm{l}]$ & 377.2 & 77.3 & 515.4 & 80.9 & 500.2 & 58.9 & 10.7 & 0.01 \\
\hline $\mathrm{GH}[\mathrm{ng} / \mathrm{ml}]$ & 0.7 & 1.0 & 40.0 & 26.9 & 27.6 & 25.8 & 8.1 & 0.01 \\
\hline IGF-1 [ng/ml] & 267.8 & 58.9 & 280.4 & 48.7 & 232.0 & 69.1 & 0.8 & 0.47 \\
\hline IGFBP-3 [ng/ml] & 3541.2 & 582.5 & 4393.0 & 2369.9 & 4440.6 & 2602.2 & 0.3 & 0.76 \\
\hline \multicolumn{9}{|c|}{ Control Group } \\
\hline $\mathrm{T}[\mathrm{nmol} / \mathrm{l}]$ & 29.9 & 5.7 & 43.5 & 12.0 & 39.9 & 10.8 & 9.3 & 0.02 \\
\hline free $\mathrm{T}[\mathrm{nmol} / \mathrm{l}]$ & 11.7 & 3.9 & 11.0 & 5.2 & 11.4 & 6.6 & 0.16 & 0.86 \\
\hline $\mathrm{C}[\mathrm{nmol} / \mathrm{l}]$ & 229.0 & 42.6 & 396.3 & 86.5 & 429.0 & 50.7 & 12.3 & 0.02 \\
\hline $\mathrm{GH}[\mathrm{ng} / \mathrm{ml}]$ & 0.4 & 0.3 & 47.1 & 14.6 & 26.0 & 11.1 & 37.1 & 0.00 \\
\hline IGF-1 [ng/ml] & 290.8 & 157.6 & 402.5 & 161.1 & 403.5 & 162.2 & 1.1 & 0.38 \\
\hline IGFBP-3 [ng/ml] & 3324.3 & 1750.7 & 4092.5 & 1423.8 & 3722.3 & 439.3 & 0.7 & 0.52 \\
\hline
\end{tabular}

Table. 5

Cardiac biomarkers in cyclists with left ventricle hypertrophy (LVH) and without left ventricle hypertrophy (CG) before (rest) and immediately post (max) incremental and continuous exercise.

\begin{tabular}{|c|c|c|c|c|c|c|c|c|}
\hline \multirow{2}{*}{ Variables } & \multicolumn{4}{|c|}{ Incremental exercise (IncEx) } & \multicolumn{4}{|c|}{ Continuous exercise (ConEx) } \\
\hline & Rest & Max & $\mathrm{F}$ & $p$ & Rest & Max & $\mathrm{F}$ & $p$ \\
\hline \multicolumn{9}{|c|}{ LVH Group } \\
\hline NT-proBNP [pg/ml] & $57.5(49.8)$ & $59.7(53.1)$ & 0.9 & 0.99 & $33.5(13.3)$ & $56.6(26.5)$ & 1.1 & 0.42 \\
\hline CK-MB [U/L] & $10.3(3.3)$ & $11.0(4.7)$ & 0.4 & 0.93 & $10.5(3.4)$ & $11.5(1.8)$ & 0.1 & 0.85 \\
\hline IMA [IU/ml] & $28.9(17.8)$ & $25.1(8.9)$ & 1.5 & 0.88 & $17.8(10.3)$ & $19.3(5.9)$ & 0.4 & 0.99 \\
\hline cTnT [pg/ml] & $92.8(75.9)$ & $144.9(99.0)$ & 2.2 & 0.74 & $72.0(37.0)$ & $100.9(91.9)$ & 2.12 & 0.91 \\
\hline $\mathrm{H}-\mathrm{FABP}[\mathrm{ng} / \mathrm{ml}]$ & $8.9(2.6)$ & $7.6(3.6)$ & 1.1 & 0.64 & $9.3(3.9)$ & $7.3(3.2)$ & 0.5 & 0.31 \\
\hline \multicolumn{9}{|c|}{ Control Group } \\
\hline NT-proBNP [pg/ml] & $86.9(57.9)$ & $100.1(42.5)$ & 0.9 & 0.71 & $82.4(67.1)$ & $81.3(61.3)$ & 1.1 & 0.99 \\
\hline CK-MB [U/L] & $7.2(1.2)$ & $7.9(2.9)$ & 0.4 & 0.99 & $11.0(4.6)$ & $12.0(6.2)$ & 0.1 & 0.85 \\
\hline IMA [IU/ml] & $29.5(14.7)$ & $22.0(3.0)$ & 1.5 & 0.77 & $26.1(15.5)$ & $19.7(3.3)$ & 0.4 & 0.72 \\
\hline cTnT [pg/ml] & 70.7 (48.9) & $93.6(62.7)$ & 2.2 & 0.45 & $102.3(61.7)$ & $170.4(88.9)$ & 2.2 & 0.49 \\
\hline H-FABP [ng/ml] & $10.1(3.5)$ & $6.7(0.5) *$ & 1.1 & 0.05 & $10.6(0.8)$ & $11.0(2.0) \#$ & 0.5 & 0.98 \\
\hline
\end{tabular}

${ }^{*} p<0.05$ significant differences between Max vs. Rest

$\# p<0.05$ significant differences between LVH vs. CG 

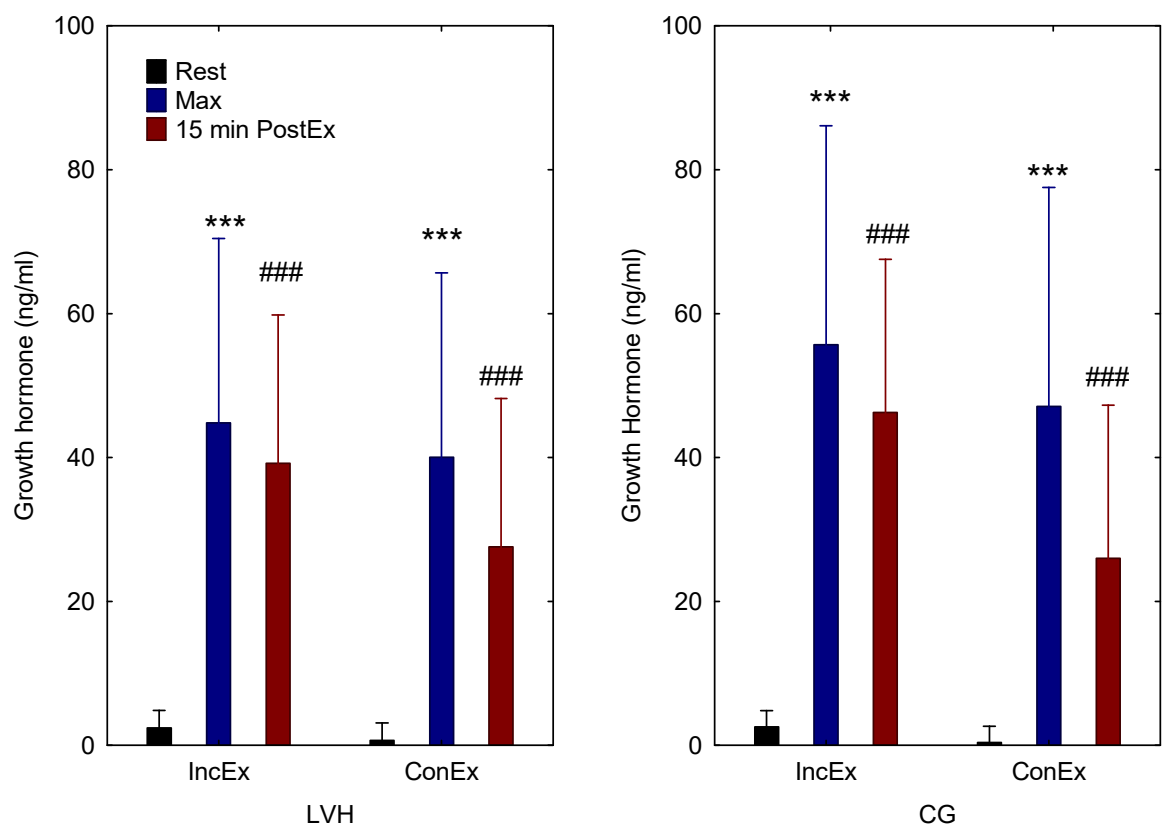

Figure 1

Growth hormone levels in cyclists with left ventricle hypertrophy (LVH), cyclists without left ventricle hypertrophy (CG) before (Rest), immediately post (Max) and after $15 \mathrm{~min}$ post (15 min PostEx) incremental (IncEx) and continuous exercise (ConEX).

${ }_{* * *} p<0.001$ Max vs Rest, \#\#\#p<0.001 15 min PostEx vs Rest

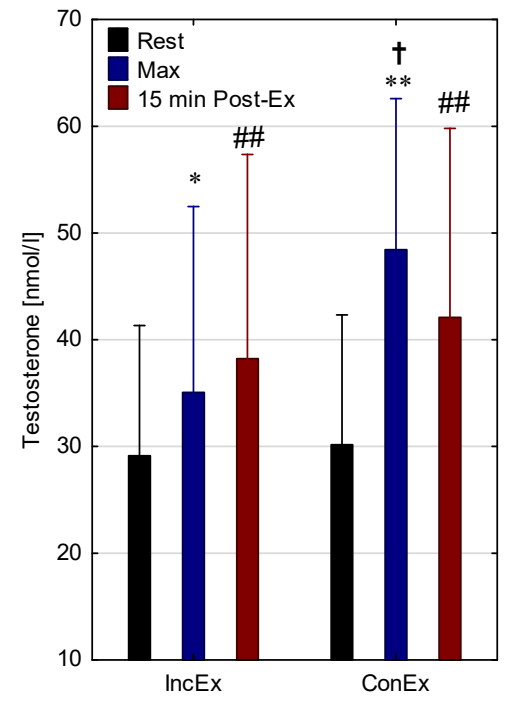

LVH

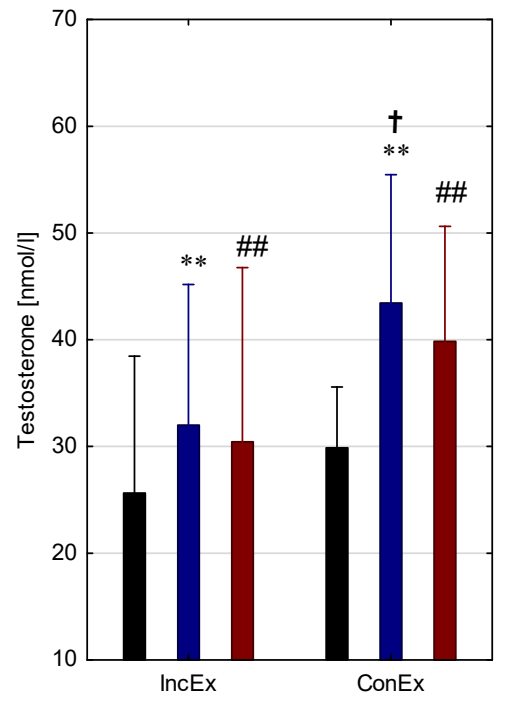

CG

Figure 2

Testosterone level in cyclists with left ventricle hypertrophy (LVH), cyclists without left ventricle hypertrophy (CG) before (Rest), immediately post (Max) and after 15 min post (15 min PostEx) incremental (IncEx) and continuous exercise (ConEX). ${ }^{*} p<0.05$ Max vs Rest, ${ }^{* *} p<0.01$ Max vs Rest, \#\#p<0.01 15 min Post-Ex vs Rest, $+p<0.05$ ConEx vs IncEx 


\section{Discussion}

Strenuous physical effort induces
structural and functional cardiovascular responses to exercise as well as activation of signaling pathways responsible for these adaptations. In our study intensive endurance training also induced high aerobic performance in cyclists (61.1 vs. $62.0 \mathrm{ml} / \mathrm{kg} / \mathrm{min}$, in the $\mathrm{LVH}$ group and the $C G$, respectively). It is worth noticing that, despite similar training experience in the studied cyclists, not all demonstrated cardiac remodeling, clearly suggesting adaptive, physiological, heart hypertrophy (Sharma, 2003). It is also interesting to note that endurance training of the LHV group did not increase the inotropic function of $\mathrm{LV}$ in our population, although a significant influence of LVH on SV, LVEF, and HR was demonstrated in previous research (Pelliccia et al., 2018).

Knowledge of the mechanisms regulating processes of cardiac remodeling in response to endurance training indicated an important role of endocrine and paracrine factors (Bleumink et al., 2005; Janikowska et al., 2014; Neri Serneri et al., 2001). The support for the impact of the endocrine system comes from data collected during ultraendurance studies, such as long distance cycling and ultramarathon races. Previous studies indicated that the elevation of serum anabolic hormones, apparently caused by muscle work, might play a crucial role in stimulating physiological heart hypertrophy in athletes (Maillet et al., 2013; Neri Serneri et al., 2001; Vega et al., 2008). Our data also confirmed differences in IGF-1 and GH levels in response to the incremental exercise protocol and in steroid hormone responses to exercise in cyclists with $\mathrm{LVH}$ relative to the CG. Post-exercise changes in testosterone concentration, lower post-exercise cortisol, and a positive correlation between $\mathrm{T} / \mathrm{C}$ and freeT levels and LVM in the LVH group indicated that all of these likely stimulate physiological LVH. Physical exercise is a strong stimulus for the endocrine system, and the hormonal response to exercise is regulated by several factors, including intensity, an individual level of training, duration, and the type of exercise (Anderson et al., 2016; Hackney et al., 2016).

In our study, cyclists with LVH demonstrated higher post exercise testosterone levels recorded in response to ConEx compared to
IncEx and significantly higher serum $\mathrm{T}$ levels were observed in post IncEx T levels in the LVH group compared to the CG. The anabolic and anticatabolic activity of testosterone is vital for exercise metabolism: it increases the synthesis of structural proteins, protects them against excessive catabolism, and supports the restoration of glycogen resources after exercise. Testosterone, IGF-1, and GH are known to be involved in pressure-overload induced cardiac growth. Several studies have demonstrated that activation of cardiac androgen receptors due to exogenous testosterone administration and endogenous secretion may stimulate myocardial and ventricular hypertrophy, under both trainingrelated (Żebrowska et al., 2017) and pathologic conditions (Mettauer et al., 2001; Payne et al., 2004). Our study revealed higher post-exercise serum $\mathrm{T}$ concentrations in the $\mathrm{LVH}$ group compared to the CG group, suggesting that testosterone might be involved in cardiac hypertrophy in experienced cyclists with LVH. The positive correlation observed between the free $\mathrm{T}$ and $\mathrm{T} / \mathrm{C}$ ratio, as well as LVMI may suggest that the hypertrophic effect observed in our cyclists was stimulated also by higher affinity of free testosterone. This could be supported by previous data which have shown that endurance training may result in up-regulation of myocardial androgen receptors, thereby increasing the effectiveness of testosterone action (Heineke et al., 2006).

Although previous studies identified a significant stimulatory influence of IGF-1 on LVH in athletes (Ner Serneri et al., 2001; Żebrowska et al., 2009), our data showed no significant differences in either IGF-1 and IGF-1/ IGFBP-3 ratio between cyclists with and without LVH. It has been shown that high intensity exercise increases serum IGF-1 compared to baseline levels in LVH subjects, suggesting that the signaling pathway of IGF-1 has a significant role in physiological cardiac hypertrophy because of its ability to regulate several cellular processes in the heart. On the other hand, it has been recently noted that the resting level of IGF-1 has a little effect on cardiac enlargement in endurancetrained adolescents (Rundqvist et al., 2019).

There is limited explanation regarding the mechanism that determines the hypertrophy and myocardium function in response to training. 
It seems that exercise induces a higher concentration of IGF-1 in relation to IGFBP-3, both at rest and during exercise in athletes with $\mathrm{LVH}$, suggesting that the effect of the IGF-1 free fraction could be greater in these individuals. It is estimated that approximately $85-95 \%$ of total IGF1 form a complex (150 kDa) with IGFBP-3 and an acid-labile subunit-ALS, however, an important role in the regulation of IGF-1 metabolism in the muscle depends on whether it is in its free or unbound form. Therefore, it is thought that the higher molar ratio of IGF-1/IGFBP-3 due to exercise-induced IGFBP-3 proteolysis may contribute to anabolic effects of exercise by increasing free IGF-1 bioavailability (Bleumink et al., 2005; Schwarz et al., 1996).

In our study, exercise-induced higher $\mathrm{GH}$ levels and IGF-1 secretions were positively related to exercise intensity during incremental exercise suggesting that high intensity exercise may be more beneficial for modulation of serum IGF-1 levels compared with low intensity continuous effort (Eliakim, 2010; Schwarz et al., 1996) in cyclists. Furthermore, no significant effect of LVH was observed in baseline IGF-1 levels as well as the baseline and post exercise IGF-1/IGFBP-3 ratio suggesting no significant influence on bioavailability of free IGF-1 in the LVH group and the CG. However, incremental exercise seems more beneficial considering long-term effects than the continuous training program in cyclists. Due to the lack of an association between increased left ventricular dimensions and baseline and postexercise serum IGF-1 and GH levels in the LVH group in our study, we may speculate that the autocrine impact of locally synthesized IGF-1 and/or the hemodynamic mechanism are of greater importance for physiological heart hypertrophy than endocrine levels of this growth factor (Heineke et al., 2006; Troncoso et al., 2014).

In this study, an impact of incremental and continuous exercise on the range of standard (e.g. NT-proBNP, cTnT, CK-MB) and novel (IMA, $\mathrm{H}-\mathrm{FABP}$ ) cardiac biomarkers was analyzed as we hypothesized that it would allow for early detection of myocardium dysfunction differences in cyclists with and without LVH (Shave et al., 2010). The main findings of this study were that the baseline and post-exercise serum concentrations of cardiac biomarkers were normal and no cardiovascular abnormalities were detected neither in the LVH group nor in cyclists with normal heart dimensions. Relatively few studies have been devoted to evaluating the complex biomarkers of cardiac' muscle in cyclists. However, a few studies comparing cardiac biomarker levels before and after ultra-endurance physical effort suggested the time-dependent increase in NT-proBNP levels, hsTnT, and CKMB activity associated with hypoxia-induced myocardium injury and wall stretch (CarranzaGarcia et al., 2011; Serrano Osteriz et al., 2013). The kinetics of these markers were differentially regulated during long distance cycling races (Corseti et al., 2012; Serrano Osteriz et al., 2013). A significant elevation of post-race CK-MB, myoglobin, highly sensitive troponin (hs-TnT) and NT-proBNP levels compared to the pre-race levels was noted in youth cyclists participating in a 177-km cycling race (Serrano Osteriz et al., 2013). It should be emphasized that our study confirmed a previous finding of Chan-Dewer et al. (2013) that high-intensity cycling trials under controlled laboratory conditions were associated with non-significant or sporadic increases in cardiac biomarkers as a sign of the exerciseinduced myocardium overload. The greatest importance of the cardiac biomarkers release in response to long-term exercise could be useful for both rapid confirmation and exclusion of myocardium injury in cyclists (Corsetti et al., 2012).

\section{Conclusions}

In summary, endurance training of cyclists caused physiological cardiac adaptations evidenced by echocardiographic variables and normal levels of cardiac biomarkers. Incremental and continuous exercise led to significant increases in testosterone and growth hormone concentrations and a moderate increase in insulin growth factor levels. Importantly, high-intensity incremental exercise seems to induce beneficial cardiac adaptations via significant increases in concentration of anabolic factors compared to training with constant exercise intensity. 


\section{References}

Anderson T, Lane AR, Hackney AC. Cortisol and testosterone dynamics following exhaustive endurance exercise. Eur J Appl Physio, 2016; 116(8): 1503-9

Bleumink GS, Schut AFC, Sturkenboom MCJM, Janssen JAMJL, Witteman JCM, van Duijn CM, Hofman A, Stricker BHCh. A promoter polymorphism of the insulin-like growth factor-I gene is associated with left ventricular hypertrophy. Heart, 2005; 91(2): 239-240 doi: 10.1136/hrt.2003.019778

Carranza-García LE, George K, Serrano-Ostáriz E, Casado-Arroyo R, Caballero-Navarro AL, Legaz-Arrese A. Cardiac biomarker response to intermittent exercise bouts. Int J Sports Med, 2011; 32: 327-331

Chan-Dewar F, Gregson W, Whyte G, Gaze D, Waterhouse J, Wen J, George K. Do the effects of high intensity $40 \mathrm{~km}$ cycling upon left ventricular function and cardiac biomarker during recovery vary with time of day? J Sports Sci, 2013; 31(4): 414-423

Corsetti R, Lombardi G, Barassi A, Lanteri P, Colombini A, D’Eril GM, Banfi G. Cardiac indexes, cardiac damage biomarkers and energy expenditure in professional cyclists during the Giro d'Italia 3-weeks stage race. Bioch Med, 2012; 22(2): 237-46

Devereux RB, Reichek N. Echocardiographic determination of left ventricular mass in man. Anatomic validation of the method. Circulation, 1977; 55: 613-618

Eliakim A, Nemet D. Exercise training, physical fitness and the growth hormone-insulin-like growth factor-1 axis and cytokine balance. Med Sport Sci, 2010; 55: 128-140

Hackney AC, Davis HC, Lane AR. Growth hormone-insulin-like growth factor axis, thyroid axis, prolactin, and exercise. Front Horm Res, 2016; 47: 1-11

Heineke J, Molkentin JD. Regulation of cardiac hypertrophy by intracellular signaling pathways. Nature Reviews Molecular. Cell Biology, 2006; 7: 589-600

Huang C, Buchanan LD, Gordon RL, Scherman MJ, Razzaq J, White K. Increased insulin-like growth factor-I gene expression precedes left ventricular cardiomyocytes hypertrophy in rapidly-hypertrophying rat model system. Cell Biochem Function, 2003; 221: 355-361

Janikowska G, Kochańska - Dziurowicz A, Żebrowska A, Bijak A, Kimsa M. Adrenergic response to maximum exercise of trained road cyclists. J Hum Kinet, 2014; 40: 103-111

Kim J, Wende AR, Sena S, Theobald HA, Soto J, Sloan C. Insulin-like growth factor I receptor signaling is required for exercise-induced cardiac hypertrophy. Molec Endocrin, 2008; 22(11): 2531-2543

Levy D, Savage DD, Garrison RJ, Anderson KM, Kannel WB, Castelli WP. Echocardiographic criteria for left ventricular hypertrophy: The Framingham heart study. Am J Cardio, 1987; 59: 956-960

LeGoff C, Kaux JF, Goffaux S, Calvalier E. Cardiac biomarkers and cycling race. J Sports Sci Med, 2015; 14(2): $475-476$

Maillet M, van Berlo JH, Molkentin JD. Molecular basis of physiological heart growth: fundamental concepts and new players. Nat Rev Mol Cell Biol, 2013; 14(1): 38-48

Mettauer B, Geny B, Landsdorf-Wolf E, Charloux A, Zhao QM, Heitz-Naegelen B, Epailly E, Lampert E, Levy F. Exercise training with a heart device: a hemodynamic, metabolic, and hormonal study. Med Sci Sports Exerc, 2001; 33: 2-9

Neri Serneri GG, Boddi M, Modesti PA, Cecioni I, Coppo M, Padeletti L, Michelucci A, Colella A, Galanti G. Increased cardiac sympathetic activity and insulin-like growth factor-I formation are associated with physiological hypertrophy in athletes. Circ Res, 2001; 89(11): 977-82

Nishimura T, Yamada Y, Kawai C. Echocardiographic evaluation of long-term effects of exercise on left ventricular hypertrophy and function in professional bicyclists. Circulation, 1980; 61(4): 832-40

Payne JR, Kotwinski PJ, Montgomery HE. Cardiac effects of anabolic steroids. Heart, 2004; 90(5): 473-475

Pelliccia A, Thompson PD. The genetics of left ventricular remodeling in competitive athletes. J Cardiovasc Med, 2006; 7: 267-270

Pelliccia A, Caselli S, Sharma S, Basso C, Bax JJ, Corrado D, D'Andrea A, D'Ascenzi, F, Di Paolo FM, Edvardsen T. European Association of Preventive Cardiology (EAPC) and European Association of Cardiovascular Imaging (EACVI) joint position statement: Recommendations for the indication and interpretation of cardiovascular imaging in the evaluation of the athlete's heart. Euro Heart J, 2018; 39: 1949-1969 
Rundqvist L, Engvall J, Blomstrand P, Carlsson E, Faresjö M. Resting Level of Insulin-Like Growth Factor-1 Is Not at Play in Cardiac Enlargement in Endurance-Trained Adolescents . BioMed Res Int, 2019, https://doi.org/10.1155/2019/9647964

Scheinowitz M, Kessler-Icekson G, Freimann S, Zimmermann R, Schaper W, Golomb E. Short-and long-term swimming exercise training increase myocardial insulin-like growth factor-I gene expression. $G H \mathcal{E}$ IGF Research, 2003; 13: 19-25

Schwarz AJ, Brasel J, Hintz RL, Mohan S, Cooper DM. Acute effect of brief low-and high-intensity exercise on circulating insulin-like growth factor (IGF) I, II and IGH binding protein-3 and its proteolysis in young healthy men. J Clin Endocrinol Metab, 1996; 81; 3492-3497

Serrano Ostariz S, López Ramón EM, Cremades Arroyos D, Izquierdo Álvarez S, Catalán Edo P, Baquer Sahún C, Legaz Arrese A. Post-exercise left ventricular dysfunction measured after a long-duration cycling event. BMC Res Notes, 2013; 26(6): 211. doi: 10.1186/1756-0500-6-211

Sharma S. Athlete's heart-effect of age, sex, ethnicity and sporting discipline. Exp Physiol. 2003; 88: 665-669

Shave R, Baggish A, George K, Wood M, Scharhag J, Whyte G, Gaze D, Thompson PD. Exercise induced cardiac troponin elevation: evidence, mechanisms, and implications. J Am Coll Cardiol, 2010; 56: 169176

Troncoso T, Ibarra C, Vicencio JM, Jaimovich E, Lavandero S, New insights into IGF-1 signaling in the heart. Tren Endocrin and Metabol, 2014; 25 (3): 128-37

Utomi V, Oxborough D, Whyte GP, Somauroo J, Sharma S, Shave R, Atkinson G, George K. Systematic review and meta-analysis of training mode, imaging modality and body size influences on the morphology and function of the male athlete's heart. Heart, 2013; 99(23): 1727-1733 doi: 10.1136/heartjnl-2012-303465

Vega RB, Konhilas JP, Kelly DP, Leinwand LA. Molecular mechanisms underlying cardiac adaptation to exercise. Cell Metab, 2017; 25:1012-1026

Zung A, Phillip M, Chalew SA, Palese T, Kowarski AA. Testosterone effect on growth and growth mediators of the GH-IGF-I axis in the liver and epiphyseal growth plate of juvenile rats. J Moll Endocrinol, 1999; 23: 209-221

Żebrowska A, Gąsior Z, Langfort J. Serum IGF-I and hormonal responses to incremental exercise in athletes with and without left ventricular hypertrophy. J Sports Sci Med, 2009; 8: 67-76

Żebrowska A, Sadowska-Krepa E, Jagsz S, Klapcinska B, Langfort J. Cardiac hypertrophy and IGF-1 response to testosterone propionate treatment in trained male rats. Open Life Science, 2017; 12: 120-127

\title{
Corresponding author:
}

\author{
Aleksandra Żebrowska \\ Institute of Sport Sciences, Academy of Physical Education, \\ Mikołowska Street 72a, 40-065 Katowice, Poland \\ $+486000973325$ \\ E-mail: a.zebrowska@awf.katowice.pl
}

